Document downloaded from:

http://hdl.handle.net/10251/122902

This paper must be cited as:

Baselga Moreno, S.; Martínez Llario, JC. (2018). Intersection and point-to-line solutions for geodesics on the ellipsoid. Studia Geophysica et Geodaetica. 62(3):353-363.

https://doi.org/10.1007/s11200-017-1020-z

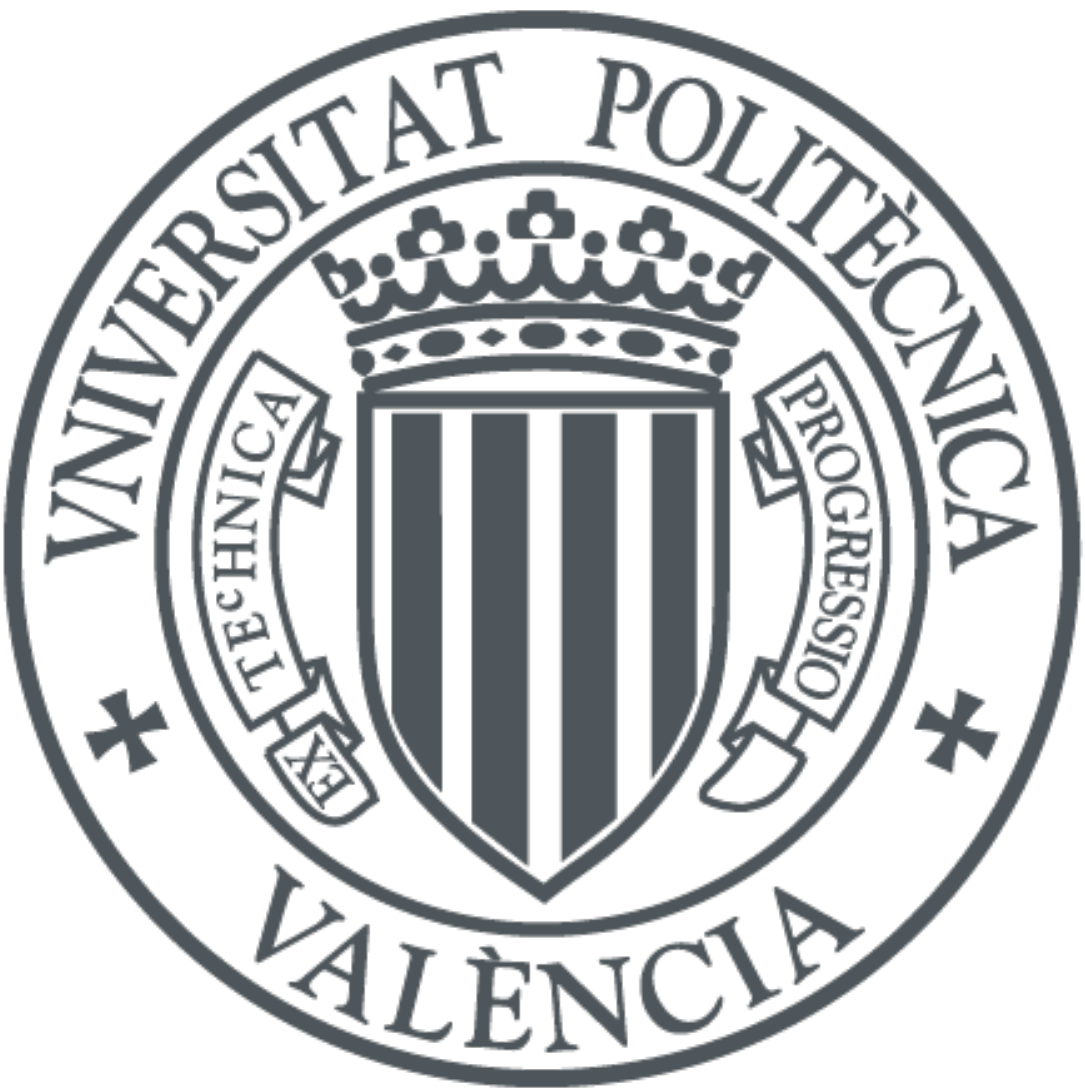

The final publication is available at

https://doi.org/10.1007/s11200-017-1020-z

Copyright Springer-Verlag

Additional Information 


\title{
Intersection and point-to-line solutions for geodesics on the ellipsoid
}

\author{
Sergio Baselga ${ }^{1, *}$ and José Carlos Martínez-Llario ${ }^{1}$ \\ ${ }^{1}$ Cartographic Engineering, Geodesy and Photogrammetry Department, Universitat \\ Politècnica de València, Camino de Vera s/n, 46022 València, Spain \\ *Corresponding author: serbamo@cgf.upv.es
}

\begin{abstract}
The paper presents two algorithms for the computation of intersection of geodesics and minimum distance from a point to a geodesic on the ellipsoid, respectively. They are based on the iterative use of direct and inverse problems of geodesy by means of their implementations with machine-precision accuracy in GeographicLib. The algorithms yield the same results as those obtained by Karney's approach based on the use of auxiliary ellipsoidal gnomonic projections with the advantage on our side that the algorithms are not limited to distances below $10000 \mathrm{~km}$. This results in our algorithm being the only general solution for the problem of minimum distance from a point to a geodesic on the ellipsoid.
\end{abstract}

\section{Keywords}

Geodesic line; Intersection; Ellipsoid; GeographicLib

\section{Introduction}

A geodesic on the ellipsoid is a curve with null geodetic curvature (i.e. null curvature on the tangent space). Given two points $A$ and $B$ on the surface on the ellipsoid, the geodesic $A B$ provides the line of minimum length that connects $A$ and $B$. Over the last centuries, geodesists have devised algorithms of increasing accuracy for solving the so-called direct and inverse problems of geodesy, that is, obtaining the coordinates of 
a point $B$ located on a geodesic starting at point $A$ with a given azimuth $\alpha_{A}$ and distance $s$ (direct problem) and obtaining the distance $s$ and azimuths at both ends $\alpha_{A}$ and $\alpha_{B}$ given the coordinates of two points $\mathrm{A}$ and $\mathrm{B}$ on the ellipsoid (inverse problem). Among the most widespread methods today we find the algorithms by Vincenty (1975), suitable for small programmable calculators, Sjöberg $(2006,2009)$ and Karney (2011a, 2013). The latter are implemented in the GeographicLib suite (Karney 2017), a library of geodetic functions developed in several programming languages that permits to take advantage of the high precision of standard computers today. State-ofthe-art open source software for spatial analysis use an implementation of GeographicLib (e.g. PostGIS Development Group 2017).

The question of intersection of geodesics has received not so much attention as the direct and inverse problems of geodesy. Sjöberg $(2002,2008,2009)$ deals with the problem directly on the ellipsoid by using numerical integration and Karney (2011a, 2011b, 2013) ingeniously solves the question by using a particular map projection (ellipsoidal gnomonic projection) in which geodesics are very nearly straight and angles are preserved at the origin. Even less attention has received the problem of minimum distance from a point to a geodesic on the ellipsoid, for which the only available procedure we find is the one based on Karney's approach using the ellipsoidal gnomonic projection (Karney 2011a, 2013), which is implemented in Karney (2011b) and JaVaWa (2017). In this paper we present new algorithms for solving these two problems. Without delving into the difficulties in dealing with the underlying systems of ordinary differential equations, their solution methods and the corresponding analyses of numerical stabilities, we resort to prior accurate implementations of the direct and inverse problems of geodesy (we recommend and will use their two implementations in GeographicLib) in order to build two easy-toimplement robust algorithms. As we will show, they yield the same results as Karney and Sjöberg's methods for intersection of geodesics and Karney's auxiliary projection method for minimum point-to-line distance except for the case of very long distances where only our methods succeed.

\section{Intersection of geodesics on the ellipsoid}


We want to derive an algorithm for computing the intersection of geodesics on the ellipsoid that is based on existing implementations of the direct and inverse problems of geodesy. We have in mind, in particular, the corresponding functions in GeographicLib, which are optimized to deliver accuracy close to machine precision. Beyond ensuring no loss of precision, we want our algorithm to be fast enough to be used not only for a single intersection but also as a possible tool in the future for more complex problems requiring multiple intersections. We describe next an iterative algorithm for the intersection of geodesics with fast convergence based on prior accurate implementations of direct and inverse problems of geodesy.

Given points $A, B, C$ and $D$ on the ellipsoid we will compute the intersection of geodesics $A B$ and $C D$. The problem is depicted in Fig. 1, where the intersection point is denoted by $X$.

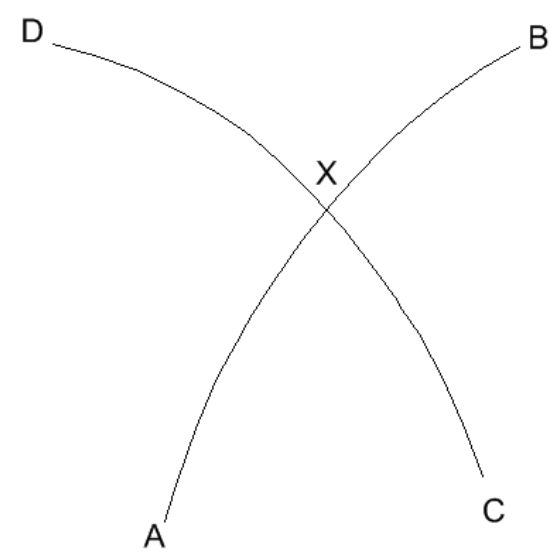

Fig. 1 Intersection of geodesics $A B$ and $C D$

A geodesic is mathematically described by a set of differential equations which are embedded in the corresponding implementations of the direct and inverse problems of geodesy. By using the implementation of the direct problem, for instance, we are able to move along the geodesic $A B$ and obtain one point at a given distance from $A$. The idea of the current method is to find an expression for the distance from $A$ to $X, s_{A X}$, that is correct for small figures and approximate enough for larger ones to yield a new point on the geodesic that is closer to the intersection. This new point will subsequently be used as base for a new iteration.

For the computation of the approximate expression of the distance between a point, say $A$, and the intersection $X$ of geodesics on the ellipsoid let us assume that $A, C$ and $X$ form a spherical triangle (where we will use a suitable value for the radius of the 
sphere $R$ as e.g. the semimajor axis of the ellipsoid). Now we resort to a rarely used formula of spherical trigonometry known as cotangent four-part formula, whose derivation can be found in Todhunter (1886), Sects. 43-44, that sets the following relation for a spherical triangle of angles $A, X, C$ (as it is common we will denote both the vertex and the angle at the vertex by the same uppercase letter) and corresponding opposite sides $a, x, c$

$\cos x \cos A=\cot c \sin x-\cot C \sin A$

By transposition we can write

$$
\tan c=\frac{\sin x}{\cos x \cos A+\cot C \sin A}
$$

and obtain the approximate expression for $s_{A X}$ that we are looking for

$$
s_{A X}=R \arctan \left(\frac{\sin \left(s_{A C} / R\right)}{\cos \left(s_{A C} / R\right) \cos A+\cot C \sin A}\right)
$$

Similarly we obtain

$$
s_{C X}=R \arctan \left(\frac{\sin \left(s_{A C} / R\right)}{\cos \left(s_{A C} / R\right) \cos C+\cot A \sin C}\right)
$$

These expressions have no significant singularities apart from the trivial case when both $A$ and $C$ are zero, i.e. both geodesics are coincident, and the entire geodesics are then the intersection. We also assume that the implementing software is able to deal with arctangents of arbitrarily large values yielding $\pm \pi / 2$.

Now Eqs. (3) and (4) do not solve the problem unless distances are sufficiently small. They provide, however, approximate values for the correct distances so that an iterative process can be started as follows.

After initial determination of azimuths $\alpha_{A B}$ and $\alpha_{C D}$ by means of the inverse geodetic problem with points $A, B$ and points $C, D$ (and considering that $\alpha_{A X}=\alpha_{A B}$ and $\alpha_{C X}=$ $\left.\alpha_{C D}\right)$ we apply the direct geodetic problem at $C$ with azimuth $\alpha_{C X}$ and the distance $s_{C X}$ obtained from Eq. (4), as well as the direct geodetic problem at $A$ with azimuth $\alpha_{A X}$ 
and the distance $s_{A X}$ obtained from Eq. (3). As shown in Fig. 2a) we obtain in the geodesic $A B$ a point $A_{2}$ which is much closer to the intersection than $A$. Similarly we obtain a point $C_{2}$ in the geodesic $C D$ which is much closer to the intersection than $C$. These points will act as new base points for the next iteration (i.e. $A$ is replaced by $A_{2}$ and $C$ by $C_{2}$ ). The new distances bring the new points, $A_{2}$ and $C_{2}$, closer to the intersection (Fig. 2b). Note that azimuths change in every iteration since the azimuth of a geodesic changes along its different base points, i.e. the values $\alpha_{A B}$ and $\alpha_{C D}$ are different in Fig. 2b) than in Fig. 2a). Iterations can be stopped when the coordinate differences between $A_{2}$ and $C_{2}$ are below the required level of accuracy. In general the method converges fast and ensures that the intersection point $X$ belongs to both geodesics (the successive application of inverse and direct problems ensures not to divert from the original geodesics).

a)

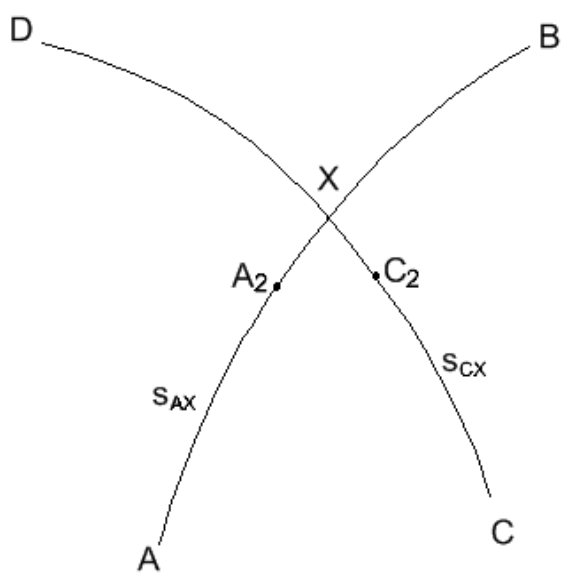

b)

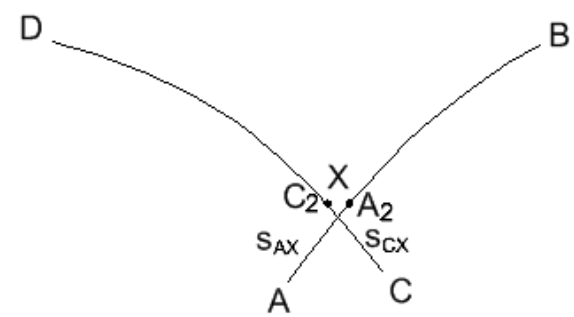

Fig. 2 Iterative procedure for the computation of the intersection point

Several notes of caution are worth mentioning here. First, the distances resulting from Eq. (3) and Eq. (4) can be negative (as it would be the case of the next iteration in Fig. 2b). Direction reversal with a positive distance shall be understood then, either by the particular implementation of the direct geodetic problem (as the corresponding function of GeographicLib does) or by inspection and modification before application of the corresponding function. This provides support also to the case where one or both lines need to be prolonged to meet the intersection. Second, apart from the intersection point $X$ shown in Fig. 1 the two geodesics intersect also in an almostantipodal point. Our method naturally yields the intersection point that is closer to $A$ 
and $C$ (assuming the arctangent function implemented returns values in the interval $\pi / 2, \pi / 2$ ), but it is easy to obtain the nearly-antipodal point provided we take only for the initial iteration the formulas

$$
\begin{aligned}
& s_{A X}=R\left[\arctan \left(\frac{\sin \left(s_{A C} / R\right)}{\cos \left(s_{A C} / R\right) \cos A+\cot C \sin A}\right)+\pi\right] \\
& s_{C X}=R\left[\arctan \left(\frac{\sin \left(s_{A C} / R\right)}{\cos \left(s_{A C} / R\right) \cos C+\cot A \sin C}\right)+\pi\right]
\end{aligned}
$$

and then we continue the course of iterations with Eqs. (3) and (4).

\subsection{Practical examples}

We start with an example where the maximum distance to the intersection point (here $s_{X B}$ ) is relatively small, $74 \mathrm{~km}$. The geodetic coordinates of the points defining geodesics $A B$ and $C D$ are $\varphi_{A}=52^{\circ}, \lambda_{A}=5^{\circ}, \varphi_{B}=51.4^{\circ}, \lambda_{B}=6^{\circ}, \varphi_{C}=51.5^{\circ}, \lambda_{C}=4.5^{\circ}$, $\varphi_{D}=52^{\circ}$ and $\lambda_{D}=5.5^{\circ}$. The course of iterations is shown in Table 1. As it can be seen our method quickly converges to coordinates for $A_{2}$ and $C_{2}$ that differ less than 0.00001 arc seconds, which constitutes our finishing criterion.

Table 1 Intersection of geodesics, example 1. Successive distances for the application of the direct problem of geodesy and corresponding coordinates of points on geodesics from $A$ (point $A_{2}$ ) and $C$ (point $C_{2}$ ).

\begin{tabular}{crrcccc}
\hline $\begin{array}{c}\text { Iteration } \\
\text { No. }\end{array}$ & \multicolumn{1}{c}{$s_{A X}(\mathrm{~m})$} & $s_{C X}(\mathrm{~m})$ & $\varphi_{A 2}$ & \multicolumn{1}{c}{$\lambda_{A 2}$} & $\varphi_{C 2}$ & \multicolumn{1}{c}{$\lambda_{C 2}$} \\
\hline 1 & 21637.0270 & 64700.0631 & $51^{\circ} 51^{\prime} 56.3972^{\prime \prime}$ & $5^{\circ} 13^{\prime} 38.8427^{\prime \prime}$ & $51^{\circ} 51^{\prime} 56.3311^{\prime \prime}$ & $5^{\circ} 13^{\prime} 38.7157^{\prime \prime}$ \\
2 & 0.0762 & 3.1832 & $51^{\circ} 51^{\prime} 56.3954^{\prime \prime}$ & $5^{\circ} 13^{\prime} 38.8456^{\prime \prime}$ & $51^{\circ} 51^{\prime} 56.3954^{\prime \prime}$ & $5^{\circ} 13^{\prime} 38.8456^{\prime \prime}$ \\
3 & 0.0000 & 0.0000 & $51^{\circ} 51^{\prime} 56.3954^{\prime \prime}$ & $5^{\circ} 13^{\prime} 38.8456^{\prime \prime}$ & $51^{\circ} 51^{\prime} 56.3954^{\prime \prime}$ & $5^{\circ} 13^{\prime} 38.8456^{\prime \prime}$ \\
\hline
\end{tabular}

We can check the correctness of the final value $\left(\varphi_{X}=51^{\circ} 51^{\prime} 56.3954^{\prime \prime}, \lambda_{X}=5^{\circ} 13^{\prime}\right.$ 38.8456") by inspection of azimuth equalities: $\alpha_{A X}=\alpha_{A B}$ (in this case $133^{\circ} 36^{\prime}$ 13.4578") and $\alpha_{B X}=\alpha_{B A}$ (in this case $314^{\circ} 23^{\prime} 18.7197^{\prime \prime}$ ) needed since the intersection point $X$ belongs to the first geodesic, and $\alpha_{C X}=\alpha_{C D}$ (in this case $50^{\circ} 45^{\prime} 17.6875^{\prime \prime}$ ) and $\alpha_{D X}=\alpha_{D C}$ (in this case $231^{\circ} 32^{\prime} 24.8830 "$ ) since the intersection point $X$ belongs to the second geodesic. All of them are dutifully fulfilled. We can also see that, as expected, in every geodetic line the azimuths from the intersection point to both ends are equal (if the line had to be prolonged) or differ exactly in $180^{\circ}$. In this example $\alpha_{X A}=133^{\circ}$ 
46' 58.1263" and $\alpha_{X B}=313^{\circ} 46^{\prime} 58.1263^{\prime \prime}$, as well as $\alpha_{X C}=51^{\circ} 19^{\prime} 32.4304^{\prime \prime}$ and $\alpha_{X D}$ $=231^{\circ} 19^{\prime} 32.4304^{\prime \prime}$.

We obtain exactly the same coordinates for the intersection by Karney's method whereas we find some discrepancies (1" in latitude, 2" in longitude) after the application of Sjöberg's (they are probably due to the numerical evaluation of the integral involved and not to the method itself).

Now we compute another example which appears in Karney (2011b) involving much longer distances (the distances to the intersection point will result in values from 1100 to $5600 \mathrm{~km})$. We take $\varphi_{A}=42^{\circ}, \lambda_{A}=29^{\circ}, \varphi_{B}=39^{\circ}, \lambda_{B}=-77^{\circ}, \varphi_{C}=6^{\circ}, \lambda_{C}=0^{\circ}, \varphi_{D}=$ $64^{\circ}$ and $\lambda_{D}=-22^{\circ}$ as coordinates defining the geodesics $A B$ and $C D$ and we have the course of iterations displayed in Table 2.

Table 2 Intersection of geodesics, example 2. Successive distances for the application of the direct problem of geodesy and corresponding coordinates of points on geodesics from $A$ (point $A_{2}$ ) and $C$ (point $C_{2}$ ).

\begin{tabular}{rrrcccc}
\hline $\begin{array}{c}\text { Iteration } \\
\text { No. }\end{array}$ & \multicolumn{1}{c}{$s_{A X}(\mathrm{~m})$} & $s_{C X}(\mathrm{~m})$ & $\varphi_{A 2}$ & $\lambda_{A 2}$ & $\varphi_{C 2}$ & $\lambda_{C 2}$ \\
\hline 1 & 3402464.8393 & 4589822.8334 & $54^{\circ} 40^{\prime} 00.1134^{\prime \prime}$ & $-13^{\circ} 45^{\prime} 42.2728^{\prime \prime}$ & $4^{\circ} 24^{\prime} 36.9467^{\prime \prime}$ & $-10^{\circ} 27^{\prime} 32.0442^{\prime \prime}$ \\
2 & 52037.0641 & 957583.2555 & $54^{\circ} 43^{\prime} 01.3449^{\prime \prime}$ & $-14^{\circ} 33^{\prime} 50.5265^{\prime \prime}$ & $54^{\circ} 37^{\prime} 33.5290^{\prime \prime}$ & $-14^{\circ} 30^{\prime} 34.4773^{\prime \prime}$ \\
3 & -11.6217 & 10723.2692 & $54^{\circ} 43^{\prime} 01.3066^{\prime \prime}$ & $-14^{\circ} 33^{\prime} 49.8807^{\prime \prime}$ & $54^{\circ} 43^{\prime} 01.3061^{\prime \prime}$ & $-14^{\circ} 33^{\prime} 49.8804^{\prime \prime}$ \\
4 & 0.0000 & 0.0152 & $54^{\circ} 43^{\prime} 01.3066^{\prime \prime}$ & $-14^{\circ} 33^{\prime} 49.8807^{\prime \prime}$ & $54^{\circ} 43^{\prime} 01.3066^{\prime \prime}$ & $-14^{\circ} 33^{\prime} 49.8807^{\prime \prime}$ \\
5 & 0.0000 & 0.0000 & $54^{\circ} 43^{\prime} 01.3066^{\prime \prime}$ & $-14^{\circ} 33^{\prime} 49.8807^{\prime \prime}$ & $54^{\circ} 43^{\prime} 01.3066^{\prime \prime}$ & $-14^{\circ} 33^{\prime} 49.8807^{\prime \prime}$ \\
\hline
\end{tabular}

We can check that the final value $\left(\varphi_{X}=54^{\circ} 43^{\prime} 1.3066^{\prime \prime}, \lambda_{X}=-14^{\circ} 33^{\prime} 49.8807^{\prime \prime}\right)$ is correct by inspection of azimuth equalities $\alpha_{A X}=\alpha_{A B}=309^{\circ} 18^{\prime} 22.4891 ", \alpha_{B X}=\alpha_{B A}$ $=47^{\circ} 44^{\prime} 07.2214^{\prime \prime}, \alpha_{C X}=\alpha_{C D}=349^{\circ} 1^{\prime} 47.4802^{\prime \prime}, \alpha_{D X}=\alpha_{D C}=154^{\circ} 29^{\prime} 46.5383 " . \mathrm{We}$ can also see that, as expected, $\alpha_{X A}=275^{\circ} 51^{\prime} 17.7681^{\prime \prime}$ and $\alpha_{X B}=95^{\circ} 51^{\prime} 17.7681^{\prime \prime}$ differ in $180^{\circ}$, as well as $\alpha_{X C}=340^{\circ} 55^{\prime} 2.9801 "$ and $\alpha_{X D}=160^{\circ} 55^{\prime} 2.9801 "$. Although the computation by Karney's method assumes that geodesics are straight lines in the ellipsoidal gnomonic projection centered in $X$ while in fact they are only "very nearly straight" (Karney 2013) we do not find any differences between our final results and those by Karney's method. By contrast, this problem does not find a reasonable result by means of Sjöberg's method. It is due to the known inability of the method to follow the geodesic beyond its vertices (Sjöberg and Shirazian 2012), since in the present example the geodesic arc $A B$ crosses the point of maximum latitude ( $\varphi$ $\left.=54^{\circ} 55^{\prime} 43^{\prime \prime}\right)$, which is in fact quite close to the intersection point. 
Finally, we compute a third example that is also intractable by Karney's method based on the gnomonic projection. Since this projection cannot represent more than one hemisphere and it has to be centered in the intersection point, the intersection problems for which at least one of the distances from the intersection point to the initial points $A, B, C$, or $D$ are longer than some $10000 \mathrm{~km}$ are impossible to solve by Karney's method. We take for instance $\varphi_{A}=35^{\circ}, \lambda_{A}=-92^{\circ}, \varphi_{B}=40^{\circ}, \lambda_{B}=52^{\circ}, \varphi_{C}=-$ $8^{\circ}, \lambda_{C}=20, \varphi_{D}=49^{\circ}$ and $\lambda_{D}=-95^{\circ}$, for which the distances to the intersection point will eventually result in values from 1100 to $11300 \mathrm{~km}$ ). Table 3 shows the course of iterations.

Table 3 Intersection of geodesics, example 3. Successive distances for the application of the direct problem of geodesy and corresponding coordinates of points on geodesics from $A$ (point $A_{2}$ ) and $C$ (point $C_{2}$ ).

\begin{tabular}{rrrcccc}
\hline $\begin{array}{c}\text { Iteration } \\
\text { No. }\end{array}$ & \multicolumn{1}{c}{$s_{A X}(\mathrm{~m})$} & \multicolumn{1}{c}{$s_{C X}(\mathrm{~m})$} & \multicolumn{1}{c}{$\varphi_{A 2}$} & \multicolumn{1}{c}{$\lambda_{A 2}$} & $\varphi_{C 2}$ & \multicolumn{1}{c}{$\lambda_{C 2}$} \\
\hline 1 & 1604865.7945 & 4348568.2893 & $47^{\circ} 31^{\prime} 26.0523^{\prime \prime}$ & $-82^{\circ} 22^{\prime} 38.4586^{\prime \prime}$ & $21^{\circ} 52^{\prime} 41.8516^{\prime \prime}$ & $-5^{\circ} 53^{\prime} 11.1551^{\prime \prime}$ \\
2 & 389269.4887 & 4565612.7810 & $50^{\circ} 24^{\prime} 28.6348^{\prime \prime}$ & $-79^{\circ} 21^{\prime} 46.4166^{\prime \prime}$ & $46^{\circ} 28^{\prime} 27.8630^{\prime \prime}$ & $-46^{\circ} 38^{\prime} 35.2232^{\prime \prime}$ \\
3 & 9744.5640 & 2273004.8317 & $50^{\circ} 28^{\prime} 44.6977^{\prime \prime}$ & $-79^{\circ} 16^{\prime} 58.1459^{\prime \prime}$ & $50^{\circ} 30^{\prime} 44.8473^{\prime \prime}$ & $-77^{\circ} 01^{\prime} 21.0483^{\prime \prime}$ \\
4 & 2.0208 & 160366.4992 & $50^{\circ} 28^{\prime} 44.7508^{\prime \prime}$ & $-79^{\circ} 16^{\prime} 58.0861^{\prime \prime}$ & $50^{\circ} 28^{\prime} 44.8137^{\prime \prime}$ & $-79^{\circ} 166^{\prime} 55.5158^{\prime \prime}$ \\
5 & 0.0000 & 50.7146 & $50^{\circ} 28^{\prime} 44.7508^{\prime \prime}$ & $-79^{\circ} 16^{\prime} 58.0861^{\prime \prime}$ & $50^{\circ} 28^{\prime} 44.7508^{\prime \prime}$ & $-79^{\circ} 16^{\prime} 58.0861^{\prime \prime}$ \\
6 & 0.0000 & 0.0000 & $50^{\circ} 28^{\prime} 44.7508^{\prime \prime}$ & $-79^{\circ} 16^{\prime} 58.0861^{\prime \prime}$ & $50^{\circ} 28^{\prime} 44.7508^{\prime \prime}$ & $-79^{\circ} 16^{\prime} 58.0861^{\prime \prime}$ \\
\hline
\end{tabular}

We obtain the result $\varphi_{X}=50^{\circ} 28^{\prime} 44.7508^{\prime \prime}, \lambda_{X}=-79^{\circ} 16^{\prime} 58.0861^{\prime \prime}$ and azimuth equalities $\alpha_{A X}=\alpha_{A B}=27^{\circ} 0^{\prime} 50.0492^{\prime \prime}, \alpha_{B X}=\alpha_{B A}=330^{\circ} 57^{\prime} 3.3917^{\prime \prime}, \alpha_{C X}=\alpha_{C D}=$ 319 57' 19.8704", $\alpha_{D X}=\alpha_{D C}=75^{\circ} 46^{\prime} 33.9244^{\prime \prime}$. We can also see that, as expected, $\alpha_{X A}=35^{\circ} 44^{\prime} 35.7125^{\prime \prime}$ and $\alpha_{X B}=215^{\circ} 44^{\prime} 35.7125^{\prime \prime}$ differ in $180^{\circ}$, as well as $\alpha_{X C}=$ $267^{\circ} 48^{\prime} 9.5053^{\prime \prime}$ and $\alpha_{X D}=87^{\circ} 48^{\prime} 9.5053^{\prime \prime}$. In this case, Sjöberg's method is unable to find the correct solution since the intersection is beyond the vertex of geodesic $C D$ when computed from $C$. We can however use Sjöberg's method and attain a result with rather small discrepancies with respect to ours $\left(0.1^{\prime \prime}\right.$ both in latitude and longitude) and 0.5 " of maximum discrepancy in the azimuth check if we interchange points $C$ and $D$, or, in other words, if we compute the intersection point from $D$ not $C$ (so that the vertex of the geodesic is not reached before the intersection). At any rate, Sjöberg's method demonstrates to be not always applicable, in contrast to ours.

\section{Minimum point-to-line distance on the ellipsoid}


The problem now is to find the minimum distance from a point $P$ to a geodesic $A B$ on the ellipsoid (Fig. 3). We can devise an algorithm based on the iterative use of the implementations of the direct and inverse problems of geodesy plus the necessary condition of intersection at right angles (Patrikalakis et al. 2009).

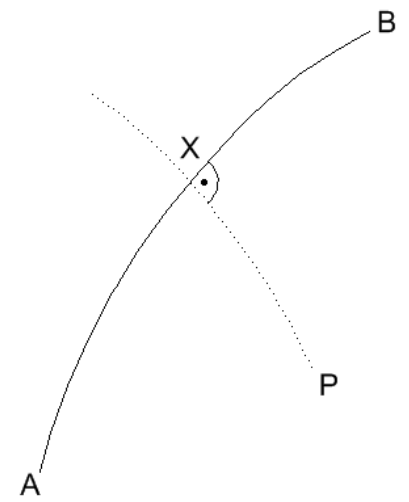

Fig. 3 Minimum point-to-line distance

Again the idea is to use approximate expressions for the distances from $A$ to $X, s_{A X}$, and $P$ to $X, s_{P X}$, that are correct for small figures and approximate enough for larger ones to yield a new point on the geodesic $A B$ that is closer to the intersection. This new point will subsequently be used as base for a new iteration.

For the computation of the approximate expressions for distances let us assume that points $A, P$ and $X$ form a spherical triangle, one that has a right angle $X$, where we will use a suitable value for the radius of the sphere $R$ (e.g. the semimajor axis of the ellipsoid).

The sine rule for angles $A, X\left(90^{\circ}\right)$ and corresponding opposite sides $a, x$ reads

$$
\sin a=\sin x \sin A
$$

$s_{P X}=R \arcsin \left[\sin \left(s_{A P} / R\right) \sin A\right]$

The use of one of Napier's analogies for the spherical triangle of angles $A, P, X\left(90^{\circ}\right)$ and corresponding opposite sides $a, p, x$ permits us to write

$\tan \frac{p}{2}=\frac{\sin \frac{90^{\circ}+A}{2}}{\sin \frac{90^{\circ}-A}{2}} \tan \frac{x-a}{2}$ 
$s_{A X}=2 R \arctan \left(\frac{\sin \frac{90^{\circ}+A}{2}}{\sin \frac{90^{\circ}-A}{2}} \tan \frac{s_{A P}-s_{P X}}{2 R}\right)$

Eqs. (8) and (10) are not approximate enough unless the distances are sufficiently small, so an iterative process must be started as follows.

Using the implementation of the inverse problem of geodesy we determine the distance $s_{A P}$ as well as azimuths $\alpha_{A P}$ and $\alpha_{A B}$. Angle $A$ is the difference of these azimuths. By means of Eq. (8) we obtain an approximate value for the distance $s_{P X}$ only to be introduced in Eq. (10) so that an approximate value for $s_{A X}$ can be obtained. Now we use the implementation of the direct problem of geodesy to compute a point $A_{2}$ along the geodesic $A B$ located at distance $s_{A X}$ from $A$, which will act as base point for the next iteration (i.e. $A$ is replaced by $A_{2}$ ), just the same as we did in the first method (Fig. 2). The difference now is that we do not know the azimuth of the geodesic $X P$ so we cannot iterate $P$. After several iterations the differences between successive coordinates of $A_{2}$ are below the required level of accuracy (or, equivalently, the distance $s_{A X}$ can be regarded zero for our level of accuracy) and the iterations can be finished.

The method converges fast to the solution of minimum distance from point $P$ to geodesic $A B$ - note that we were not interested in the other possible larger value of the arcsin function in Eq. (8) - supports line prolonging and has no significant singularities (it yields the correct solution even if the point $P$ is in the geodesic $A B$ ).

Next we apply the algorithm to some examples and compare the results with those that can be obtained by Karney (2011b) by means of use of the auxiliary gnomonic projection.

\subsection{Practical examples}

Let us start with an example where the point-to-line distance is relatively small, some $24 \mathrm{~km}$. The geodetic coordinates of the points defining the geodesic $A B$ and point $P$ are $\varphi_{A}=52^{\circ}, \lambda_{A}=5^{\circ}, \varphi_{B}=51.4^{\circ}, \lambda_{B}=6^{\circ}, \varphi_{P}=52^{\circ}$ and $\lambda_{P}=5.5^{\circ}$. The course of iterations is shown in Table 4. As it can be seen, our method converges to the final 
value in just three iterations (our finishing condition is that successive coordinates for $A_{2}$ differ less than 0.00001 arc seconds).

Table 4 Point to geodesic, example 1. Successive coordinates of point $A_{2}$ and corresponding distances

\begin{tabular}{cccr}
\multicolumn{4}{c}{$s_{A X}}$. \\
$\begin{array}{cccr}\text { Iteration } \\
\text { No. }\end{array}$ & $\varphi_{A 2}$ & $\lambda_{A 2}$ & \multicolumn{1}{c}{$s_{A X}(\mathrm{~m})$} \\
\hline 1 & $51^{\circ} 50^{\prime} 45.9212^{\prime \prime}$ & $5^{\circ} 15^{\prime} 37.5426^{\prime \prime}$ & 24784.2886 \\
2 & $51^{\circ} 50^{\prime} 45.9212^{\prime \prime}$ & $5^{\circ} 15^{\prime} 37.5426^{\prime \prime}$ & -0.0002 \\
3 & $51^{\circ} 50^{\prime} 45.9212^{\prime \prime}$ & $5^{\circ} 15^{\prime} 37.5426 "$ & 0.0000 \\
\hline
\end{tabular}

We ensure that the solution $\varphi_{X}=51^{\circ} 50^{\prime} 45.9212^{\prime \prime}, \lambda_{X}=5^{\circ} 15^{\prime} 37.5426^{\prime \prime}$ is correct by checking right-angle conditions (in this case $\alpha_{X A}=313^{\circ} 48^{\prime} 31.4766^{\prime \prime}, \alpha_{X P}=43^{\circ} 48^{\prime}$ 31.4766" and $\left.\alpha_{X B}=133^{\circ} 48^{\prime} 31.4766 "\right)$ along with the condition that $X$ is on both geodesics (which was indeed part of our implementation of intersection of geodesics: $\alpha_{A X}=133^{\circ} 36^{\prime} 13.4578^{\prime \prime}$ and $\alpha_{A B}=133^{\circ} 36^{\prime} 13.4578^{\prime \prime}$, as well as $\alpha_{B X}=314^{\circ} 23^{\prime}$ $18.7191^{\prime \prime}$ and $\left.\alpha_{B A}=314^{\circ} 23^{\prime} 18.7191 "\right)$. The results are the same following Karney's approach.

Our second example is taken from Karney (2011b) and involves a point-to-line distance much longer (around $1000 \mathrm{~km}$ ). With $\varphi_{A}=42^{\circ}, \lambda_{A}=29^{\circ}, \varphi_{B}=39^{\circ}, \lambda_{B}=-77^{\circ}$, $\varphi_{P}=64^{\circ}$ and $\lambda_{P}=-22^{\circ}$ we have the course of iterations shown in Table 5.

Table 5 Point to geodesic, example 2. Successive coordinates of point $A_{2}$ and corresponding distances

\begin{tabular}{cccr}
\multicolumn{4}{c}{$s_{A X}}$. \\
$\begin{array}{cccc}\text { Iteration } \\
\text { No. }\end{array}$ & $\varphi_{A 2}$ & $\lambda_{A 2}$ & $s_{A X}(\mathrm{~m})$ \\
\hline 1 & $54^{\circ} 55^{\prime} 42.7066^{\prime \prime}$ & $-21^{\circ} 56^{\prime} 18.1328^{\prime \prime}$ & 3928857.7554 \\
2 & $54^{\circ} 55^{\prime} 42.7134^{\prime \prime}$ & $-21^{\circ} 56^{\prime} 14.2477^{\prime \prime}$ & -69.1851 \\
3 & $54^{\circ} 55^{\prime} 42.7134^{\prime \prime}$ & $-21^{\circ} 56^{\prime} 14.2477^{\prime \prime}$ & 0.0000 \\
\hline
\end{tabular}

Again it is checked that the solution $\varphi_{X}=54^{\circ} 55^{\prime} 42.7134^{\prime \prime}, \lambda_{X}=-21^{\circ} 56^{\prime} 14.2477^{\prime \prime}$ fulfills right-angle conditions $\left(\alpha_{X A}=89^{\circ} 49^{\prime} 31.0947^{\prime \prime}, \alpha_{X P}=359^{\circ} 49^{\prime} 31.0943^{\prime \prime}\right.$ and $\left.\alpha_{X B}=89^{\circ} 49^{\prime} 31.0947^{\prime \prime}\right)$, belongs to geodesic $A B\left(\alpha_{A X}=309^{\circ} 18^{\prime} 22.4891^{\prime \prime}\right.$ and $\alpha_{A B}=$ $309^{\circ} 18^{\prime} 22.4891^{\prime \prime}$, as well as $\alpha_{B X}=47^{\circ} 44^{\prime} 7.2214^{\prime \prime}$ and $\left.\alpha_{B A}=47^{\circ} 44^{\prime} 7.2214^{\prime \prime}\right)$ and is exactly coincident with the one in Karney (2011b).

Finally, we compute an example that is intractable by Karney's method based on the gnomonic projection due to its limitation to one hemisphere (i.e. requires that all distances from the projection center, point $X$, be below $10000 \mathrm{~km}$ ). With $\varphi_{A}=42^{\circ}, \lambda_{A}$ 
$=29^{\circ}, \varphi_{B}=-35^{\circ}, \lambda_{B}=-70^{\circ}, \varphi_{P}=64^{\circ}$ and $\lambda_{P}=-22^{\circ}$ we have the course of iterations shown in Table 6.

Table 6 Point to geodesic, example 3. Successive coordinates of point $A_{2}$ and corresponding distances $s_{A X}$.

\begin{tabular}{cccr}
\hline $\begin{array}{c}\text { Iteration } \\
\text { No. }\end{array}$ & $\varphi_{A 2}$ & $\lambda_{A 2}$ & \multicolumn{1}{c}{$s_{A X}(\mathrm{~m})$} \\
\hline 1 & $37^{\circ} 58^{\prime} 39.9186^{\prime \prime}$ & $18^{\circ} 20^{\prime} 53.7147^{\prime \prime}$ & 1012443.9063 \\
2 & $37^{\circ} 58^{\prime} 41.2237^{\prime \prime}$ & $18^{\circ} 20^{\prime} 56.6280^{\prime \prime}$ & -81.6969 \\
3 & $37^{\circ} 58^{\prime} 41.2237^{\prime \prime}$ & $18^{\circ} 20^{\prime} 56.6280^{\prime \prime}$ & 0.0000 \\
\hline
\end{tabular}

The solution $\varphi_{X}=37^{\circ} 58^{\prime} 41.2236^{\prime \prime}, \lambda_{X}=18^{\circ} 20^{\prime} 56.6279 "$ verifies right-angle conditions $\left(\alpha_{X A}=60^{\circ} 29^{\prime} 33.8570 ", \alpha_{X P}=330^{\circ} 29^{\prime} 33.8570^{\prime \prime}\right.$ and $\alpha_{X B}=240^{\circ} 29^{\prime}$ $\left.33.8570^{\prime \prime}\right)$ and belongs to geodesic $A B\left(\alpha_{A X}=247^{\circ} 21^{\prime} 11.9791 "\right.$ and $\alpha_{A B}=247^{\circ} 21^{\prime}$ 11.9791", as well as $\alpha_{B X}=56^{\circ} 53^{\prime} 14.0821^{\prime \prime}$ and $\left.\alpha_{B A}=56^{\circ} 53^{\prime} 14.0821 "\right)$. As mentioned before, the resulting distance $s_{X B} \approx 12200 \mathrm{~km}$ is longer than $10000 \mathrm{~km}$, what makes the problem intractable by Karney's method and, as far as we know, by any other method that has been published so far.

\section{Conclusions}

We have presented two algorithms for solving the problems of intersection of geodesics and minimum distance from a point to a geodesic on the ellipsoid, respectively. They can be applied to all range of distances: from local-scale problems (a few $\mathrm{km}$ ) to global-scale ones (even more than $10000 \mathrm{~km}$ ). Being iterative in nature, and the second algorithm also resorting to the implementation of the first, their computational cost is dependent on the distances involved. They represent a competitive implementation with the currently standard approach by Karney based on the use of an auxiliary ellipsoidal gnomonic projection, with the advantage on our side that Karney's method is inapplicable for distances longer that $10000 \mathrm{~km}$. This results in our algorithm being the only possibility for accurately solving the general problem of minimum distance from a point to a geodesic on the ellipsoid.

\section{Acknowledgments}


The authors are sincerely thankful to the editor and two anonymous reviewers for their detailed comments and suggestions that helped to considerably improve the methods developed in the manuscript as well as its readability.

\section{References}

JaVaWa, 2017. JAVAWA GPS Tools. (online at http://www.javawa.nl/coordcalc_en.html. Accessed 23 January 2017).

Karney C.F.F., 2011a. Geodesics on an ellipsoid of revolution. Tech rep, SRI International. (online at http://arxiv.org/abs/1102.1215v1 Accessed 15 December 2016).

Karney C.F.F., 2011b. Intersection between two geodesic lines. (online at https://sourceforge.net/p/geographiclib/discussion/1026621/thread/21aaff9f. Accessed 15 February 2017).

Karney C.F.F., 2013. Algorithms for geodesics. J. Geod., 87(1), 43-55.

Karney C.F.F., 2017. GeographicLib, version 1.47. (online at http://geographiclib.sourceforge.net)

Patrikalakis N.M., Maekawa T., Cho W., 2009. Shape interrogation for computer aided and manufacturing. (online at http://web.mit.edu/hyperbook/Patrikalakis-Maekawa-Cho/mathe.html. Accessed 18 April 2017).

PostGIS Development Group, 2017. PostGIS 2.3.3dev Manual. (online at http://postgis.net/docs/index.html. Accessed 23 February 2017).

Sjöberg L.E., 2002. Intersections on the sphere and ellipsoid. J. Geod., 76, 115-120.

Sjöberg L.E., 2006. Direct and indirect geodetic problems on the ellipsoid. ZfV, 131, $35-39$.

Sjöberg L.E., 2008. Geodetic intersection on the ellipsoid . J. Geod., 82(9), 565-567. 
Sjöberg L.E., 2009. New solutions to classical geodetic problems on the ellipsoid. In: Sideris M.G. (Ed.) Observing our changing earth, International Association of Geodesy Symposia 133, 781-784, Springer-Verlag Berlin Heidelberg.

Sjöberg L.E. and Shirazian M, 2012. Solving the direct and inverse geodetic problems on the ellipsoid by numerical integration. J. Surv. Eng., 138(1), 9-16.

Todhunter I., 1886. Spherical trigonometry, $5^{\text {th }}$ Ed., MacMillan and Co. (available at http://www.gutenberg.org/ebooks/19770. Accessed 2 May 2017)

Vincenty T., 1975. Direct and inverse solutions of geodesics on the ellipsoid with application of nested equations. Surv. Rev., 23(176), 88-93 [addendum: Surv Rev 23(180), 294 (1976)]. 